\title{
On the "elementary" solution of Laplace's Equation
}

\author{
By H. S. Ruse, Edinburgh University. \\ (Received 5th June 1930. Read 7th June 1930.)
}

\section{§1. Introduction.}

Hadamard defines" the "elementary solution" of the general linear partial differential equation of the second order, namely

$$
F(u) \equiv \sum_{i, k=1}^{n} A_{i k} \frac{\partial^{2} u}{\partial x_{i} \partial x_{k}}+\sum_{i=1}^{n} B_{i} \frac{\partial u}{\partial x_{i}}+C u=0,
$$

$\left(A_{i k}, B_{i}, C\right.$ being functions of the $n$ variables $x_{1}, x_{2}, \ldots, x_{n}$, which may be regarded as coordinates in a space of $n$ dimensions), to be one of those solutions which are infinite to as low an order as possible at a given point and on every bicharacteristic through that point. ${ }^{2} \mathrm{He}$ then proceeds to find formulae for the elementary solution of equation (1), his result being as follows:

Let $\left(H_{i k}\right)$ be the matrix reciprocal to $\left(A_{i k}\right)$, and consider the Riemannian space whose metric is specified by

$$
d s^{2}=\sum_{i, k} H_{i k} d x_{i} d x_{k} \text {. }
$$

Let $\Gamma$ denote the square of the geodesic distance between the point $\left(x_{i}\right)$ and a fixed point $\left(q_{i}\right)$ of this space. Then if $n$ be odd, the elementary solution of ( 1 ) is given by

where

$$
u=\Gamma^{-\frac{1}{2}(n-2)}\left[U_{0}+U_{1} \Gamma+U_{2} \Gamma^{2}+\ldots+U_{r} \Gamma^{r}+\ldots\right]
$$

$$
U_{0}=k \exp \left[-\int_{0}^{s} \frac{1}{4 s}\{F(\Gamma)-C \Gamma-2 n\} d s\right]
$$

$k$ being a certain constant, $s=\sqrt{ } \Gamma$, the integral being taken along the are of the geodesic from $\left(q_{i}\right)$ to $\left(x_{i}\right)$. The functions $U_{r}(r>0)$ are then determined by the recurrence-formula

$$
U_{r}=-\frac{U_{0}}{(4 r-2 n+4) s^{r}} \int_{0}^{s} \frac{s^{r-1}}{U_{0}} F\left(U_{r-1}\right) d s
$$

${ }^{3}$ Lectures on Cauchy's Problem in Linear Partial Differential Equations (Yale, 1923), p. 70 , et seq.

2 The solutions satisfying this condition differ only in the values of arbitrary constants, the elementary solution being obtained by choosing these according to a certain rule. For the purpose of this paper it suffices to say that the elementary solution is the one which reduces to $u=1 / r$, where $r=\sqrt{ }\left\{(x-\bar{x})^{2}+(y-\bar{y})^{2}+(z-\bar{z})^{2}\right\}$, when the differential equation (1) is of the particular form $\nabla^{2} V=0$. 
A similar formula holds when $n$ is even, but involves a term in $\log \Gamma$.

The object of the present paper is to establish a formula for the elementary solution for the particular case in which equation (1) is the tensor generalisation, with respect to the metric (2), of the ordinary Laplace's equation. The result obtained lacks the generality of Hadamard's, but may be of interest on account of its comparative simplicity and because a single formula holds whether $n$ is odd or even.

\section{§2. Laplace's Equation in Tensor Form.}

Let

$$
d s^{2}=g_{\mu \nu} d x^{\mu} d x^{\nu}, \quad(\mu, \nu=1,2, \ldots, n),
$$

define the metric ${ }^{1}$ of a general Riemannian space of $n$ dimensions.

If $V$ be any scalar and $V_{\mu \nu}$ its second covariant derivative, viz.

$$
V_{\mu \nu}=\frac{\partial^{2} V}{\partial x^{\mu} \partial x^{\nu}}-\{\mu \nu, \alpha\} \frac{\partial V}{\partial x^{a}},
$$

then the partial differential equation of which we seek the elementary solution is

$$
g^{\mu \nu} V_{\mu \nu}=0 .
$$

(When $n=3$ and $d s^{2}=d x^{2}+d y^{2}+d z^{2}$, this reduces to the ordinary Laplace's Equation $\nabla^{2} V=0$ ).

Let $\Omega$ be one half the square of the geodesic distance ${ }^{2}$ between the point $\left(x^{i}\right) \equiv\left(x^{1}, x^{2}, \ldots, x^{n}\right)$ and the fixed point $\left(\bar{x}^{i}\right) \equiv\left(\bar{x}^{1}, \bar{x}^{2}, \ldots, \bar{x}^{n}\right)$. Thus

$$
\Omega=\frac{1}{2} s^{2}
$$

where $s$ is the length of the arc of the geodesic joining $\left(x^{i}\right)$ and $\left(\bar{x}^{i}\right)$.

$\Omega$ is a function of the $x$ 's and of the $\bar{x}$ 's. We shall write $\Omega_{\mu}$ for $\partial \Omega / \partial x^{\mu}, \quad \Omega_{(\mu)}$ for $\partial \Omega / \partial \bar{x}^{\mu}, \quad \Omega_{\mu(\nu)}$ for $\partial^{2} \Omega / \partial x^{\mu} \partial \bar{x}^{\nu}$. Further, $\bar{g}_{\mu \nu}, \bar{g}^{\mu \nu}$ will be used respectively to denote the values at $\left(\bar{x}^{i}\right)$ of $g_{\mu \nu}, g^{\mu \nu}$, while $\bar{g}$ will represent the value at this point of the determinant $g=\left\|g_{\mu \nu}\right\|$.

1 The summation convention is adopted throughout. The notation of the succeeding paragraphs will differ to some extent from that of Hadamard, in order that it should be brought into conformity with the notation now usual in the Tensor Calculus.

2 Thus if in $\S 1\left(q_{i}\right)$ is taken at $(\bar{x})$, we have $\Omega=\frac{1}{2} \Gamma$. 
Let $J$ denote the determinant $\left\|\Omega_{\mu(\nu)}\right\|$. Then we shall prove the following theorem:

The elementary solution of the partial differential equation $g^{\mu \nu} V_{\mu \nu}=0$ is given by

$$
V=A \int_{g^{\frac{1}{2}}}^{s} \frac{J}{\bar{g}^{\frac{1}{2}}} \frac{d s}{s^{n-1}}+B,
$$

where $A, B$ are suitable constants, and the integral is taken along an arc of the geodesic joining $\left(\bar{x}^{i}\right)$ to $\left(x^{i}\right)$.

It should be remarked that $\left(\bar{x}^{i}\right)$ must not itself be a point of the arc of integration, for if it were the integral would in general be divergent. Further, the constant $B$ must be so chosen that the solution is unaltered by an interchange of the the $x^{i}$ with the $\bar{x}^{i}$. The actual value given to $A$ is not of fundamental importance.

\section{§3. Proof of the theorem of $\S 2$.}

It is a known fact that by transferring to a normal coordinatesystem $\left(y^{i}\right)$, the equations of any geodesic through $\left(\bar{x}^{i}\right)$ can be put in the form ${ }^{1}$

$$
y^{i}=a^{i} s,
$$

where the constants $a^{i}$ are the values at $\left(\bar{x}^{i}\right)$ (which is the origin of the normal coordinates) of $d x^{i} / d s$ for the geodesic in question. Thus

$$
a^{i}=\frac{d \bar{x}^{i}}{d s} \text {. }
$$

Components of tensors corresponding to the $y$-coordinate-system will be denoted by the affixing of an asterisk. For example, $* \Omega_{\mu}$ will denote the vector $\partial \Omega / \partial y^{\mu}$.

By (3) and (8), $\bar{g}_{\mu \nu} a^{\mu} a^{\nu}=1$. Multiplying by $\frac{1}{2} s^{2}$ and using (5), we get

$$
\Omega=\frac{1}{2} \bar{g}_{\mu \nu} y^{\mu} y^{\nu} \text {. }
$$

Hence

$$
\frac{\partial \Omega}{\partial y^{\mu}}=\bar{g}_{\mu \nu} y^{\nu}
$$

that is,

$$
* \Omega_{\mu}=* g_{\mu \nu} y^{\nu},
$$

since $^{2}$

$$
\bar{g}_{\mu \nu} y^{\nu}={ }^{*} g_{\mu \nu} y^{\nu} \text {. }
$$

1 The $y^{i}$ are the normal variables of Lipschitz: Hadamard, loc. cit., p. 87. A full account of them is given by Veblen, Invariants of Quadratic Differential Forms (Cambridge Tract no. 24, 1927), ch. VI.

$\overline{2}$ Veblen, loc. cit., ch. VI (14.8). 
Raising the suffix $\mu$ in (10),

Hence

$$
* \Omega^{\mu}=y^{\mu} \text {. }
$$

Contracting,

$$
\begin{aligned}
* \Omega_{\nu}^{\mu} & =\frac{\partial * \Omega^{\mu}}{\partial y^{\nu}}+*\{\alpha \nu, \mu\} * \Omega^{\alpha} \\
& =\delta_{\nu}^{\mu}+*\{\alpha \nu, \mu\} y^{\alpha} .
\end{aligned}
$$

or $^{1}$, by (7),

$$
* \Omega_{\mu}^{\mu}=n+\frac{1}{2} y^{\alpha} \frac{\partial}{\partial y^{a}}(\log * g)
$$

$$
* \Omega_{\mu}^{\mu}=n+\frac{1}{2} s \frac{d}{d s}(\log * g) .
$$

Again, it has been shown ${ }^{2}$ that

$$
y^{\mu}=-\bar{g}^{\mu \alpha} \Omega_{(\alpha)}
$$

the repetition of $\alpha$ implying a summation.

By (5), ${ }^{*} \Omega^{\mu}=s^{*} s^{\mu}$, and it quickly follows from $(10),(9)$ and (5), that

$$
*_{s_{\mu}} *_{s^{\mu}}=1
$$

And it further follows from (11) and (13) that

$$
*_{\mu}^{\mu}=\frac{n-1}{s}+\frac{1}{2} \frac{d}{d s}\left(\log *_{g}\right) .
$$

We are now in a position to solve the partial differential equation (4). Transferring to normal coordinates, the equation becomes

But

$$
*^{\mu \nu}\left[\frac{\partial^{2} V}{\partial y^{\mu} \partial y^{\nu}}-*\{\mu \nu, a\} \frac{\partial V}{\partial y^{\alpha}}\right]=0 \text {. }
$$

Substituting in (15), we get

$$
\frac{\partial^{2} V}{\partial y^{\mu} \partial y^{\nu}}=*^{*} s_{\mu} s_{\nu} \frac{d^{2} V}{d s^{2}}+\frac{\partial^{*} s_{\mu}}{\partial y^{\nu}} \frac{d V}{d s} .
$$

$$
*_{s_{\mu}} *_{s^{\mu}} \frac{d^{2} V}{d s^{2}}+*_{s_{\mu}^{\mu}} \frac{d V}{d s}=0
$$

and hence, by (13) and (14),

$$
\frac{d^{2} V}{d s^{2}}+\left[\frac{n-1}{s}+\frac{1}{2} \frac{d}{d s}(\log * g)\right] \frac{d V}{d s}=0
$$

1 This equation is essentially the same as Hadamard's, loc. cit., p. 91 (37).

2 In a paper by the author to appear shortly in Proc. London Math. Soc. 
ON THE " elementary" solution of Laplace's Equation 139

Multiplying by the integrating factor ${ }^{*} g^{\frac{1}{2}} \mathcal{s}^{n-1}$, we quickly get the solution

$$
V=K \int^{s} \frac{1}{* g^{\frac{1}{2}}} \frac{d s}{s^{n-1}}+B,
$$

$K, B$ being arbitrary constants.

Now ${ }^{*} g^{\frac{1}{2}}$ is a scalar density, hence

$$
\begin{aligned}
* g^{\frac{1}{2}} & =g^{\frac{1}{2}} \frac{\partial\left(x^{i}\right)}{\partial\left(y^{j}\right)} \\
& =g^{\frac{1}{2}} / \frac{\partial\left(y^{i}\right)}{\partial\left(x^{j}\right)},
\end{aligned}
$$

by a well-known property of Jacobians.

But

$$
\begin{aligned}
\frac{\partial\left(y^{i}\right)}{\partial\left(x^{j}\right)} & =\left\|\frac{\partial y^{\mu}}{\partial x^{\nu}}\right\| \\
& =\left\|-\bar{g}^{\mu \alpha} \Omega_{\nu(a)}\right\| \quad \text { by }(12), \\
& =(-1)^{n} \bar{g}\left\|\Omega_{\nu(\alpha)}\right\| \\
& =(-1)^{n} \bar{g} J .
\end{aligned}
$$

Substituting from (17) and (18) in (16), and putting $A$ for the arbitrary constant $(-1)^{n} K \bar{g}^{3}$, we get

$$
V=A \int^{s} \frac{J}{g^{\frac{1}{2}}-\frac{1}{g^{2}}} \frac{d s}{s^{n}-1}+B,
$$

the result stated; $\bar{g}^{-\frac{1}{2}}$ is of course a constant, being a function of the $\bar{x}^{i}$ only. Since we have made no supposition regarding $n$, the solution holds whether $n$ be odd or even. 